\title{
Unsteady Low-Reynolds Number Flow Control in Different Regimes
}

\author{
Young-Chang $\mathrm{Cho}^{* 1}$ and Wei Shyy ${ }^{\dagger 1,2}$ \\ ${ }^{I}$ Department of Aerospace Engineering, University of Michigan, Ann Arbor, MI, 48109 \\ ${ }^{2}$ Hong Kong University of Science and Technology, Clear Water Bay, Hong Kong
}

\begin{abstract}
Low-Reynolds number wings are susceptible to flow separation due to high viscous effects. Combined with wind gusts, the aerodynamic performance can highly fluctuate. In this study, we continue to investigate the open-loop and closed-loop control strategies aimed at mitigating lift fluctuations due to free-stream unsteadiness for a SD7003 airfoil at the chord Reynolds number of 1000. The flow control framework is based on the dielectric barrier discharge (DBD) actuator and a penalty-driven adaptive law incorporating generic system identification methods. The Strouhal number and reduced frequency of the freestream oscillation defines multiple disturbance regimes, where topological changes in liftdrag profile, stall vortex shedding, and multi-harmonic oscillations are characterized. The control system effectively reduces the lift fluctuations due to the disturbance-driven unsteady vortex dynamics, offering improved understanding of effective control mechanisms.
\end{abstract}

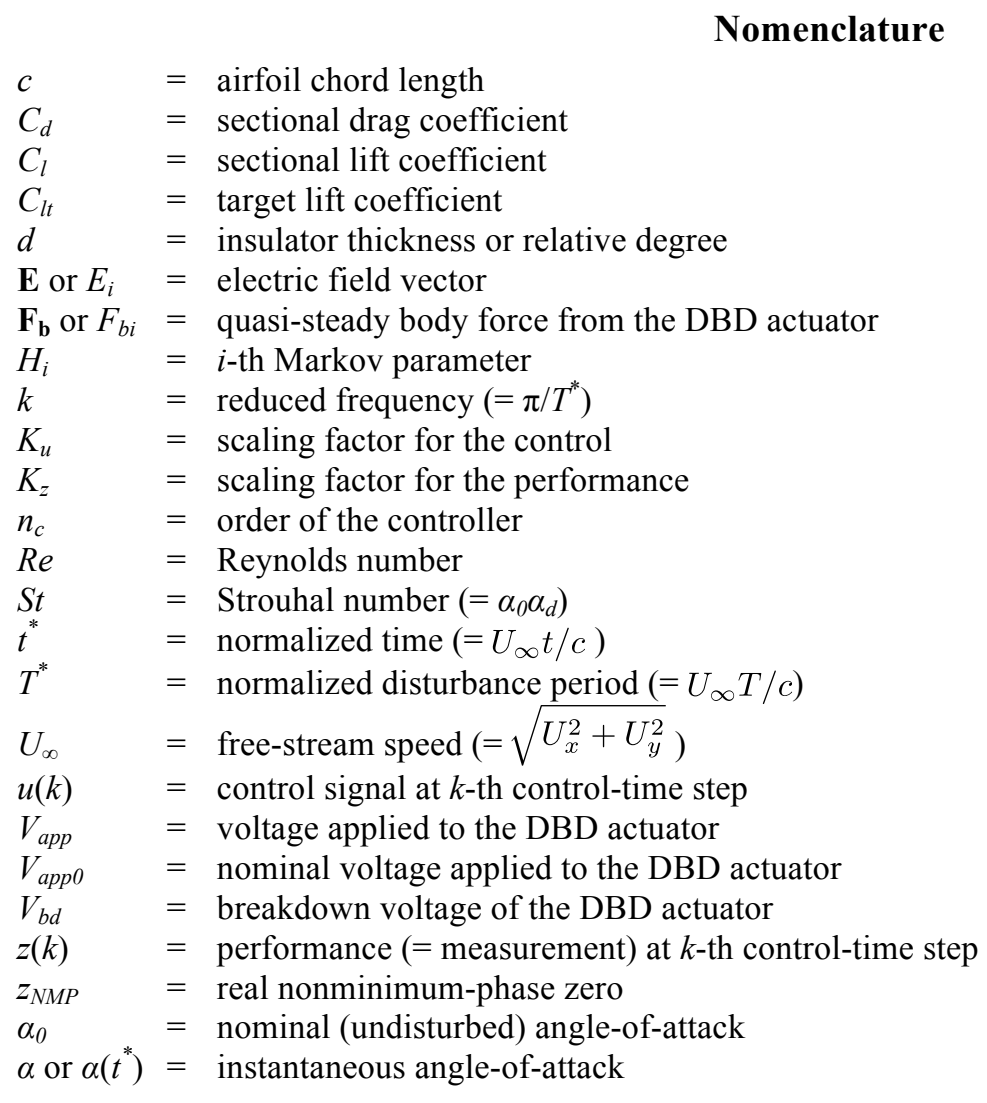

\footnotetext{
* Postdoctoral Research Fellow, AIAA Member.

${ }^{\dagger}$ Provost \& Chair Professor, Department of Mechanical Engineering, Hong Kong University of Science and Technology, also Adjunct Professor, Department of Aerospace Engineering, University of Michigan, AIAA Fellow.
} 
$=$ static stall angle-of-attack

$=$ stall angle slope

$=$ amplitude of sinusoidal disturbance in $\mathrm{y}$-directional free-stream velocity

$=$ learning rate of the adaptive controller

$=$ static stall interval

\section{Introduction}

U NSTEADY aerodynamics at low-Reynolds numbers, usually $R e=O\left(10^{3}-10^{5}\right)$, can be originated by several reasons: inherent unstable dynamics, time-varying flow or moving geometry, and turbulent flow. Considering the length and time scales of unsteadiness that are comparable to the system's characteristic scales, it is often important to exploit unsteady aerodynamics. For example, wake instability leading to vortex shedding from a bluff body has been widely studied for a wide range of Reynolds numbers for various interests in structure/vehicle designs and heat management. Aerodynamic bodies experiencing changes in free-stream, which are common in practice, can result in significant performance losses: dynamic stall is one of the major issues in low-pressure turbine blades. In small air vehicles, with either fixed or moving wings, aerodynamic performance of lifting surfaces is highly dependent on unsteady flow evolutions.

Unsteady aerodynamics associated with dynamic stall has been extensively investigated for rotorcraft design. In particular, the role of separation vortex on aerodynamic loads is recognized as a key dynamic feature along with the prescribed airfoil/wing motions. For example, as shown in Ref. 1, a pitching airfoil during dynamic stall often involves leading edge vortex formation, growth, and convection in addition to boundary layer separation and reattachment. Meanwhile, the unsteady lift curve exhibits delayed stall, lift overshoot, and hysteresis, which are known to be dependent on the rate of angle-of-attack change near the stall angle.

Although the flows around helicopter blades are usually subject to compressibility effect and occur at highReynolds numbers, similar dynamic stall processes are observed at lower Reynolds numbers. At lower Reynolds numbers, such as $R e=O\left(10^{3}-10^{5}\right)$, dynamic stall often involves the formation, growth and burst of laminar separation bubble. For a plunging airfoil in Ref. 2, while flow structures and aerodynamic loads are consistent, an earlier but more coherent stall vortex shedding and reduced aerodynamic load peaks are observed as the Reynolds number decreases. As shown in Ref. 3, while the vortex shedding frequency of a stationary airfoil is similar to bluff bodies, i.e., exhibiting a consistent Strouhal number, an abrupt change to a higher value is observed at $R e=100,000$. On the other hand, flow responses are highly dependent on the conditions of forced unsteadiness. In Ref. 4, multiple vortex generation modes are observed at different Strouhal numbers for an oscillating airfoil. For dynamic stall, the order of stall onset and the instant maximum angle-of-attack can affect the type of stall ${ }^{5}$.

In order to alleviate undesirable load fluctuations, boundary layer, shear layer, wake or instabilities therein can be manipulated by actuation. For example, a steady or periodic boundary layer excitation can increase stall angle and reduce aerodynamic load hysteresis ${ }^{6}$. However, aerodynamic forces during dynamic stall cannot be stabilized simply by suppressing coherent vortex structures or preventing flow separation. In this case, the instantaneous flow field should be resolved and changed systematically to achieve regulated pressure and/or friction forces. Furthermore, unsteady flow separation can be used to enhance aerodynamic force generation, heat exchange, and mixing at low-Reynolds numbers. For example, unsteady vortex dynamics of moving wings can increase timeaveraged lift or thrust beyond steady-state performance ${ }^{7}$. Unsteady vortex flow can be also adopted to enhance heat transfer in electronic devices ${ }^{8}$.

When a flow system encounters off-design flow conditions, such as wind gusts, unsteady wake, and intermittent interference with near bodies, the loss of aerodynamic performance is expected. Similarly to the flow around moving bodies, such as pitching or plunging airfoil, the changes in local flow direction and/or magnitude can induce dynamic flow separation, unsteady shear-layer, and vortex evolution, as well as their interactions. However, unlike moving bodies, where the influence of prescribed motions is of interest, in most cases free-stream unsteadiness is irregular and has wide bandwidth. Such uncertainty makes it difficult to characterize the interaction between the free-stream disturbance and induced/triggered flow dynamics.

In the design of thermo-fluid systems, various flow control devices have been applied to alleviate system's performance loss at off-design conditions supported by progress in actuator and control algorithm. Active flow control adopting control strategies with positive energy expenditure intends to enhance system's operability without major parasitic effects. Furthermore, modern non-mechanical fluid devices, such as synthetic jets and plasma-based actuators, make dynamic flow manipulation more feasible. The dielectric barrier discharge (DBD) actuator, among other plasma-based devices, is promising for this purpose along with its distinct advantages: no moving parts, high bandwidth (fast response) and least complexity of installation. Although its actuation capacity is still limited, i.e., 
typical induced flow speed of $O(1) \mathrm{m} / \mathrm{s}$, the unique advantages of the $\mathrm{DBD}$ actuator justifies the recent active research on its various applications, such as lift enhancement ${ }^{9}$, stalled wing ${ }^{10}$, flow-induced noise reduction ${ }^{11,12}$, and boundary layer transition delay ${ }^{13}$.

Despite extensive efforts in the past, high-dimensionality and nonlinearity of most practical flow systems inhibit a comprehensive analysis of the system's dynamics. As a result, one of the fundamental goals in the study of unsteady aerodynamics is to understand the leading mechanisms responsible for its influence on the system's performance, such as vehicle stability, aerodynamic forces, and heat transfer. Here, an effective control scheme not only contributes to performance enhancement but also provides better understanding in unsteady aerodynamics. Unlike quasi-steady or open-loop control strategies that rely on fixed/scheduled operational conditions, a control goal that incorporates unsteady dynamics requires a closed-loop system. The relevant questions are (1) how to allocate sensors and actuators to capture key flow information and to provide sufficient control authority, respectively, (2) how to model the flow-actuator system and handle nonlinearity, and (3) how to get essential model parameters. Since most flow systems of interest are highly nonlinear, it is often infeasible to analytically predict control performance. Some reduced-order and nonlinear modeling approaches prove their effectiveness for specific cases: cylinder wake control using proper orthogonal decomposition (POD) ${ }^{14}$, separation point control using wallshear equation ${ }^{15}$, and control of lift fluctuation on a wing based on low-order linear flow and disturbance models ${ }^{16}$. However, since key dynamics that a fluid system model should account for is not straightforward in complex flows, controllers based on rigorous modeling are neither feasible nor promising. One roundabout is the application of adaptive laws, which let the control system estimate unmodeled parameters. By tuning a set of modeling parameters during operation, unaccounted physics and uncertainty in system modeling can be compensated by the adaptive process. In this case, the physical meaning of tuned parameters is of less concern.

In our previous work ${ }^{17}$, key flow physics and nonlinearity sources are discussed for a low-Reynolds number airfoil under the influence of unknown free-stream oscillations. Furthermore, the lift fluctuation is mitigated by a DBD actuator regulated by an adaptive control law, the retrospective cost adaptive controller ${ }^{18}$, which uses system parameters obtained from Markov parameters. While relying on the adaptive law that involves no disturbance model, the key parameters of the linearized system model are identified through off-line impulse or white noise tests. The controlled performance and corresponding flow fields suggest that the baseline/controlled flow features, required control effort, and control effectiveness change depending on the disturbance frequency and magnitude. Moreover, it is shown that the control system exhibits a higher target dependency and sometimes control failure under certain disturbance conditions.

In this study, the baseline/controlled flow physics and efficacy of the control system are characterized more comprehensively for the same canonical problem - lift stabilization for a single-harmonic free-stream fluctuation is considered. Specifically, the disturbance domain is categorized into multiple regimes where dominant flow physics, flow patterns, and control mechanisms differ distinctively. Disturbance criteria based on disturbance parameters - Strouhal number and reduced frequency - are introduced to assist the categorization. Major objectives of this study are (1) to understand leading unsteady aerodynamics associated with a high-angle-of-attack airfoil/wing under oscillating free-stream, (2) to probe effective lift stabilization mechanisms for different disturbance conditions, and (3) to explore the potential and limitation of linear model-based adaptive control.

The contents of this paper are four parts: in sections II and III, models for flow simulation, control theory, and system identification process are summarized; Section IV includes A) key flow physics of the open-loop system and B) disturbance regimes and closed-loop control performance.

\section{Aerodynamics and DBD Actuator Models}

For the numerical simulation of the flow-actuator system, the same models as Ref. 19 are used in this study. For clarity, they are summarized in this section.

\section{A. Fluid Dynamics Model}

The flow fields are analyzed by solving the incompressible Navier-Stokes equations using the Loci-STREAM ${ }^{20}$, a parallelized pressure-based unstructured finite volume code. Since the ion and electron states are non-equilibrium and the ion temperature is comparable to the neutral fluid, the neutral fluid is treated as being isothermal. Considering the time scale disparity between the neutral flow and the radio frequency (RF) actuator operation, the resultant body force acting on the neutral fluid is assumed to be a quasi-steady. The body force felt by the neutral flow is equivalent to the Lorentz force acting on the net charge density. For the unsteady operation of the actuator 
only the amplitude variation of the operation voltage with time scales much larger than the RF operation is considered. In index notation, the relevant conservation equations are

$$
\begin{aligned}
\frac{\partial u_{j}^{*}}{\partial x_{j}^{*}} & =0, \\
\frac{\partial u_{i}^{*}}{\partial t^{*}}+\frac{\partial\left(u_{i}^{*} u_{j}^{*}\right)}{\partial x_{j}^{*}} & =F_{b i}^{*}-\frac{\partial p^{*}}{\partial x_{i}^{*}}+\frac{1}{R e} \frac{\partial}{\partial x_{j}^{*}}\left(\frac{\partial u_{i}^{*}}{\partial x_{j}^{*}}\right),
\end{aligned}
$$

where, $i, j=1,2$, and 3 for 3 -dimensional flow. Here, $F_{b i}^{*}$ is DBD body force vector defined below, $x_{i}^{*}=x_{i} / c$ is the Cartesian position in the global coordinate system, $u_{i}^{*}=u_{i} / U_{\infty}$ is the flow velocity, $p^{*}=\left(p-p_{\infty}\right) / \rho U_{\infty}$ is the pressure, all in normalized quantities, and $R e=\rho U_{\infty} c / \mu$ is the Reynolds number ( $c$ is the wing chord length, $U_{\infty}$ is the free-stream speed, $p_{\infty}$ is the free-stream pressure, $\rho$ is the air density, and $\mu$ is the dynamic viscosity of air). The time resolution for the flow simulations is $\Delta t_{f}^{*}=U_{\infty} \Delta t_{f} / c=0.05$, where $\Delta t_{f}$ is the dimensional time resolution, while the control time step is $\Delta t_{c}^{*}=10 \Delta t_{f}^{*}$. As an example, for a chord of $5 \mathrm{~cm}$ in a free-stream speed of $0.3 \mathrm{~m} / \mathrm{s}$, which corresponds to the chord-based Reynolds number of 1000, the flow time resolution and the control time resolution are 8 milliseconds and 0.08 second, respectively.

The flow-actuator system is a SD7003 airfoil where the DBD actuator is located at $x / c=0.2$ on the suction side. The undisturbed angle-of-attack is $15^{\circ}$, which results in unsteady flow separation when there is no actuation. The free-stream disturbance is modeled as spatially uniform, temporal oscillations: a sinusoidal function of time is introduced in the $y$-direction $\mathbf{U}\left(t^{*}\right)=\left(U_{x}, U_{y}\left(1+\alpha_{d} \sin \left(2 \pi t^{*} / T^{*}\right)\right)\right)$, where $\alpha_{d}$ is the disturbance amplitude, and $T^{*}$ is the normalized disturbance period. The disturbance amplitude and period can be related to the Strouhal number $S t \triangleq\left(\alpha_{d} U_{\infty} \sin \alpha_{0}\right) / U_{\infty} \simeq \alpha_{0} \alpha_{d}$ and reduced frequency $k \triangleq \pi T^{-1} /\left(U_{\infty} / c\right)=\pi / T^{*}$.

\section{B. DBD Actuator Model}

The DBD actuator model is the simplified body-force model with a spatially bilinear electric field and constant net charge density ${ }^{21}$. As shown in Figure 1, this model prescribes localized body forces in a triangular plasma region bounded by two electrodes and the dielectric surface. The electric field distribution inside the plasma region is approximated by

$$
\begin{gathered}
\mathbf{E}^{\prime}\left(x^{\prime}, y^{\prime}, t\right)=\left(\frac{\left|\mathbf{E}^{\prime}\left(x^{\prime}, y^{\prime}, t\right)\right| k_{2}}{\sqrt{k_{1}^{2}+k_{2}^{2}}}, \frac{\left|\mathbf{E}^{\prime}\left(x^{\prime}, y^{\prime}, t\right)\right| k_{1}}{\sqrt{k_{1}^{2}+k_{2}^{2}}}\right), \\
\left|\mathbf{E}^{\prime}\left(x^{\prime}, y^{\prime}, t\right)\right|=E_{0}(t)-k_{1} x^{\prime}-k_{2} y^{\prime}, \quad E_{0}(t)=\frac{V_{a p p}(t)}{d},
\end{gathered}
$$

where $\left(x^{\prime}, y^{\prime}\right)$ is the actuator local coordinate system, $d$ is the insulator thickness, and $k_{1}$ and $k_{2}$ are the linearized slopes of the electric field (or electric field attenuation constants) in the $x^{\prime}$ and $y^{\prime}$ directions, respectively. This model is a solution of Gauss' equation with the assumptions of constant net charge density and bilinear electric field. In (4), the maximum electric field intensity $E_{0}$ is defined based on the applied voltage to the exposed electrode $V_{a p p}(t)$, which implies the amplitude modulation of the radio frequency AC voltage, and the slopes $k_{1}$ and $k_{2}$ are set to allow the breakdown voltage at the hypotenuse boundary where the electric field strength is minimum. As defined in the next section, the applied voltage is the scaled control signal that is bounded by the breakdown voltage, resulting in a time-varying body force under feedback control. As a result, this analytical-empirical model results in a body-force field acting on the fluid, given by

$$
\mathbf{F}_{b}(x, y, t)=\rho_{c} q_{c} \delta(x, y) f_{v} \Delta t_{d} \mathbf{E}(x, y, t),
$$

where $\delta(x, y)$ is 1 within the triangular region and 0 elsewhere, $f_{v}$ is the AC frequency of the voltage applied to the DBD actuator and $\Delta t_{d}$ is the discharge duty cycle, and $\mathbf{E}(x, y, t)$ is the electric field distribution (3) transformed to the global coordinate system ${ }^{21,22}$. For this study, as shown in Figure 1, the horizontal and vertical electric field dimensions are set to $0.05 c$ and $0.025 c$, respectively. 


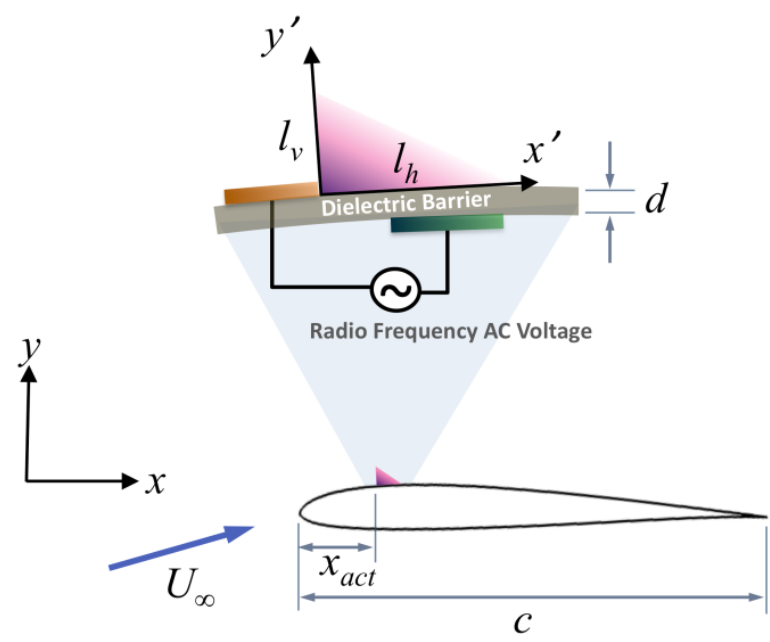

Figure 1 Schematics of the SD7003 airfoil and DBD actuator model.

The discharge duty cycle is the portion of time during which effective force generation occurs in each operation cycle. Here, it should be noted that the reduced-order DBD model (3)-(5) is meaningful when the time scale of the applied voltage is much larger than that of plasma operation.

\section{System Identification and Control}

\section{A. Retrospective Cost Adaptive Algorithm}

For the control update the same formulations of adaptive controller as Ref. 19 are used in this study, which are summarized here for clarity. The block diagram of the flow-actuator system and controller is shown in Figure 2 . The controller $G_{c}$ takes the difference between the current lift $C_{l}$ and the target lift $C_{l t}$ as the measurement, which is equal to the performance. Although it is reasonable as a target to use the nominal lift coefficient, which is the lift with nominal actuation but no disturbance, different targets are also used for the test cases because, as described with the results, the control performance is sensitive to the target lift.

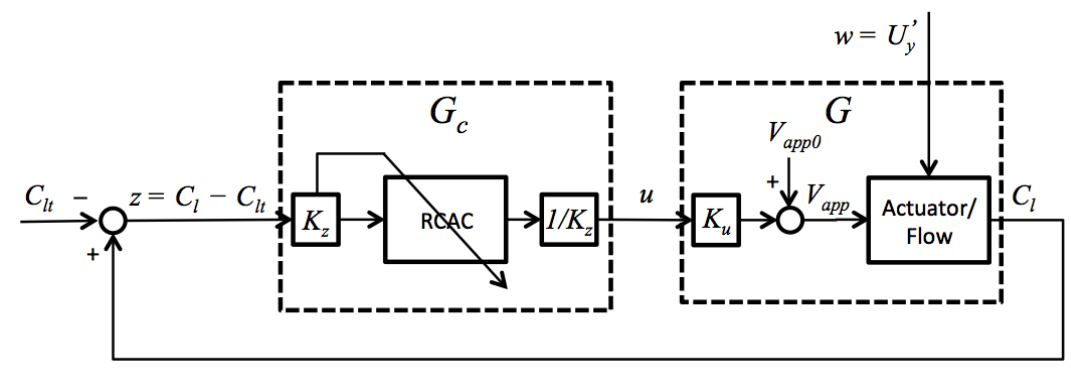

Figure 2 Block-diagram of the controller and flow-actuator system.

In order to scale variables for the controller and for the actuator, two scaling factors $K_{z}$ and $K_{u}$ are introduced, respectively. This scaling helps to keep the control and the performance to be in the same range, which can significantly affect the control performance. Furthermore, the scaling relieves the performance dependency on the tunable parameter in the controller, namely the learning rate that is introduced below. For the controller, the retrospective cost adaptive control (RCAC) algorithm ${ }^{18}$ is summarized in this section for a single-input, singleoutput control system along with some modifications for the scaling. A single-input, single-output linear discretetime system can be presented as

$$
\begin{aligned}
x(k+1) & =A x(k)+B u(k)+D_{1} w(k), \\
z(k) & =E_{1} x(k)+E_{0} w(k),
\end{aligned}
$$

where $x(k) \in \mathbb{R}^{n}, z(k) \in \mathbb{R}, u(k) \in \mathbb{R}, w(k) \in \mathbb{R}^{l_{w}}$ are the state, performance, control, and exogenous command and/or disturbance signal with $k \geq 0$. In this paper, the exogenous signal $w$ is the influence of the free-stream 
disturbance. The control objective is to minimize the performance, which is the difference between instantaneous and target aerodynamic forces, using an adaptive output feedback controller.

For the general control problem given by (6)-(7), a strictly proper time-series controller of order $n_{c}$ is given by

$$
u(k)=\sum_{i=1}^{n_{c}} M_{i}(k) u(k-i)+\sum_{i=1}^{n_{c}} N_{i}(k) z(k-i),
$$

where, for all $i=1, \cdots, n_{c}, M_{i}(k), N_{i}(k) \in \mathbb{R}$ are given by the adaptive law presented below. The control can be expressed as

$$
u(k)=\theta^{T}(k) \phi(k),
$$

where

$$
\theta(k) \triangleq\left[\begin{array}{llllll}
N_{1}(k) & \cdots & N_{n_{c}}(k) & M_{1}(k) & \cdots & M_{n_{c}}(k)
\end{array}\right]^{T}, \in \mathbb{R}^{2 n_{c}}
$$

is the controller parameter matrix, and the regressor vector $\phi(k)$ is given by

$$
\phi(k) \triangleq\left[\begin{array}{llllll}
z(k-1) & \cdots & z\left(k-n_{c}\right) & u(k-1) & \cdots & u\left(k-n_{c}\right)
\end{array}\right]^{T} \in \mathbb{R}^{2 n_{c}} .
$$

We define the extended control vector $U(k)$ by

$$
U(k) \triangleq\left[\begin{array}{c}
u(k) \\
\vdots \\
u\left(k-m_{c}\right)
\end{array}\right] \in \mathbb{R}^{m_{c}+1},
$$

where $m_{c}$ is the number of Markov parameters retained in the system parameter matrix $\bar{B}_{z u}$ that is given by Eq. (25) below. From (9), it follows that the extended control vector $U(k)$ can be written as

$$
U(k) \triangleq \sum_{i=1}^{m_{c}+1} L_{i} \theta^{T}(k-i+1) \phi(k-i+1),
$$

where

$$
L_{i} \triangleq\left[\begin{array}{c}
0_{(i-1) \times 1} \\
1 \\
0_{\left(p_{c}-i\right) \times 1}
\end{array}\right] \in \mathbb{R}^{p_{c}} .
$$

Next, define the retrospective performance

$$
\hat{z}(\hat{\theta}, k) \triangleq z(k)-\bar{B}_{z u}(U(k)-\hat{U}(\hat{\theta}, k))
$$

where $\hat{U}(\hat{\theta}, k) \triangleq K_{z} \sum_{i=1}^{p_{c}} L_{i} \hat{\theta}^{T} \phi(k-i+1), \hat{\theta} \in \mathbb{R}^{2 n_{c}}$ is an optimization variable. Note that $\hat{z}(\hat{\theta}, k)$ is obtained by modifying the performance variable $z(k)$ based on the difference between the actual past control inputs $U(k)$ and the recomputed past control inputs $\hat{U}(\hat{\theta}, k)$ assuming that $\hat{\theta}$ had been used in the past. Thus, $\hat{z}(\hat{\theta}, k)$ may be interpreted as an approximation of the performance had $\hat{\theta}$ been used in the past.

Now, consider the retrospective cost function

$$
\hat{J}(\hat{\theta}, k) \triangleq \hat{z}^{2}(\hat{\theta}, k)+\alpha_{l}(\hat{\theta}-\theta(k))^{T}(\hat{\theta}-\theta(k)),
$$

where the learning rate $\alpha_{l}$ affects the transient performance and the convergence speed of the adaptive control algorithm. Substituting (15) into (16) yields

$$
\hat{J}(\hat{\theta}, k)=\hat{\theta}^{T} A(k) \hat{\theta}+b^{T}(k) \hat{\theta}+c(k),
$$

where

$$
A(k) \triangleq D^{T}(k) D(k)+\alpha_{l} I
$$




$$
\begin{aligned}
& b(k) \triangleq 2 K_{z} D^{T}(k)\left(z(k)-\bar{B}_{z u} U(k)\right)-2 \alpha_{l} \theta(k), \\
& c(k) \triangleq K_{z}^{2}\left(z(k)-\bar{B}_{z u} U(k)\right)^{2}+\alpha_{l} \theta^{T}(k) \theta(k),
\end{aligned}
$$

and $D(k) \triangleq K_{y} \bar{B}_{z u} \sum_{i=1}^{p_{c}}\left(L_{i} \phi^{T}(k-i+1)\right)$. Here, the learning rate is defined as $\alpha_{l} \triangleq K_{z}^{2}$ for an appropriate scaling. Since $A(k)$ is positive definite, $\hat{J}(\hat{\theta}, k)$ has a unique global minimizer, which can be used for the update law

$$
\theta(k+1)=-\frac{1}{2} A^{-1}(k) b(k) .
$$

The adaptive controller (9) and (18)-(21) requires limited model information of the plant (6)-(7); however, the controller does require knowledge of $\bar{B}_{z u}$, which is constructed using estimates of the plant's relative degree, first nonzero Markov parameter, and the nonminimum-phase (NMP) zeros of the transfer function from $u$ to $z$. Since the actuation voltage $V_{a p p}$ is limited by actuator saturation at the breakdown voltage, the controller is informed of its amplitude saturation. As a result, the actual control $u(k)$ is chosen as $\max \left(u(k), u_{b d}\right)$, where $u_{b d}$ is the control that results in the breakdown voltage.

\section{B. Identification of System Parameters}

Markov parameters are the time-domain impulse response of a discrete-time linear time-invariant system, and they are very functional in system identification especially due to their uniqueness to the system. On the other hand, NMP zeros are zeros of the rational transfer function that lie outside the unit circle in the complex domain. Though the latter is sometimes called unstable zeros, they are not a measure of the system's stability. Instead, it is known that a NMP system incurs a fundamental limitation in feedback control performance ${ }^{23}$. In addition, an odd number of real NMP zeros in continuous-time systems are responsible for and related to the initial undershoot, which are also observed for the current flow-actuator system as shown in the previous study ${ }^{22}$.

In order to construct the control matrix using aforementioned system parameters, consider the transfer function from $u$ to $z$ given by

$$
G_{z u}(z) \triangleq E_{1}(z I-A)^{-1} B
$$

which can be written as

$$
G_{z u}(z) \triangleq H_{d} \frac{\beta(z)}{\alpha(z)}
$$

where the relative degree $d \geq 1$ is the smallest positive integer $i$ such that the $i$-th Markov parameter $H_{i} \triangleq E_{1} A^{i-1} B$ is nonzero, and $\alpha(z)$ and $\beta(z)$ are monic coprime polynomials. Next, let $\beta(z)$ have the factorization

$$
\beta(z) \triangleq \beta_{s}(z) \beta_{u}(z)
$$

where $\beta_{s}(z)$ is a monic polynomial of degree ns whose roots lie inside the unit circle, and $\beta_{u}(z)$ is a monic polynomial of degree nu whose roots lie on or outside the unit circle. Furthermore, we can write $\beta_{u}(z)=z^{n_{u}}+\beta_{u, 1} z^{n_{u}-1}+\cdots+\beta_{u, n_{u}-1} z+\beta_{u, n_{u}}$. Then we let $\mu=n_{u}+d$, and the resulting control matrix $\bar{B}_{z u}$ is given by

$$
\bar{B}_{z u} \triangleq H_{d}\left[\begin{array}{lllll}
0_{1 \times d} & 1 & \beta_{u, 1} & \cdots & \beta_{u, n_{u}}
\end{array}\right] \in \mathbb{R}^{1 \times p_{c}} .
$$

Note that $\bar{B}_{z u}$ is constructed using knowledge of the relative degree $d$, the first nonzero Markov parameter $H_{d}$, and the nonminimum-phase zeros of $\beta_{u}(z)$ of the transfer function from $u$ to $z$.

The first nonzero Markov parameter and NMP zeros of a linear model can be estimated by using the impulse response (i.e., Markov parameters) of the model. Our previous study showed that the flow-actuator system is a nonminimum-phase system with the relative degree of 1 , meaning that the system has a reverse initial response with one step time lag. Accordingly, based on the parameter scaling using $K_{z}=1$ and $K_{u}=10^{4}$, the first nonzero Markov parameter and the learning rate are -1 and 1 , respectively, and only NMP zeros are estimated for system identification. 


\section{Results and Discussion}

\section{A. Open-Loop Flow Physics under Unsteady Free-Stream}

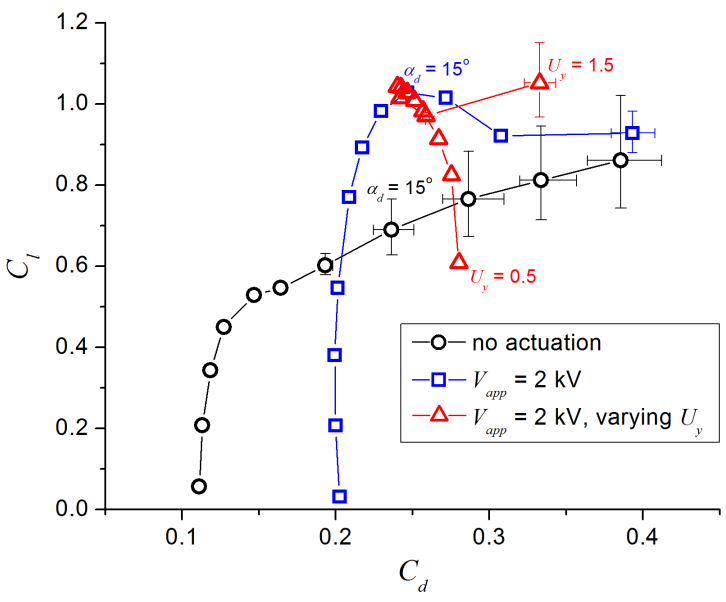

(b)

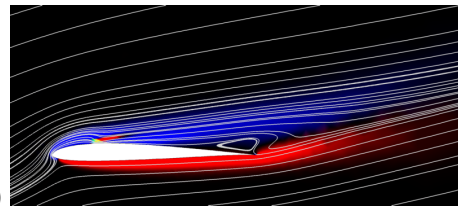

(c)

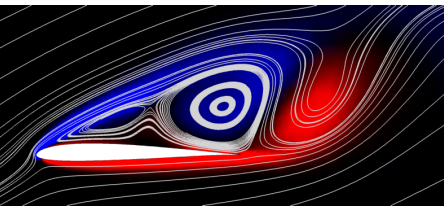

(a)

(d)

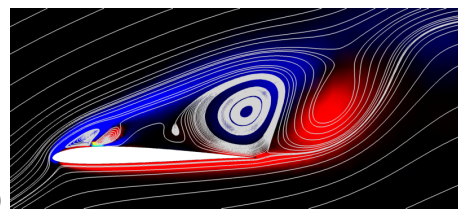

Figure 3 Steady free-stream and flow fields (streamlines and vorticity contours - blue: clockwise, red: counter-clockwise): (a) lift-drag relation (error bars indicate the maximum and minimum of each), (b) flow field snapshot at $\alpha=15^{\circ}, V_{a p p}=2 \mathrm{kV}$ (nominal flow), (c) at the moment of maximum lift for $\alpha=21^{\circ}$ without actuation, (d) at the moment of maximum lift for $U_{y}{ }^{\prime}=0.5 U_{y}, V_{a p p}=2 \mathrm{kV}$.

Without the DBD actuation, the lift increase slows down due to flow separation as the angle-of-attack increases and exhibits unsteady vortex shedding as shown in Figure 3a) with the normalized frequency of $k=2.1$ at the angle of attack of $13^{\circ}$, which decreases to $k=1.4$ for $21^{\circ}$. When the nominal actuation of $V_{a p p}=2 \mathrm{kV}$ is applied, the drag at low angles of attack increases substantially due to the additional skin friction introduced by the wall jet flow downstream of the actuator. At the same time, however, the high-angle-of-attack aerodynamic performance is enhanced due to reduced flow separation and delayed wake instability. In addition, the nominal actuation introduces noticeable static stall around $17^{\circ}$, which is absent for the baseline lift curve.

Considering the changes in the $y$-directional free-stream as disturbance, both the angle of attack and free-stream speed are subject to change. As a result, when the $y$-directional free-stream changes by $-50-+50 \%$, which corresponds to the effective angle-of-attack in the range of $8.1-21.9^{\circ}$, the lift-drag relation develops into a profile different from the aforementioned drag polar as shown in Figure 3a). In fact, this difference is mainly caused by the way aerodynamic force coefficients are evaluated. For the case with the change in $U_{y}$, the same angle-of-attack and free-stream speed as the baseline flow are used, which overvalues lift coefficient and undervalues drag coefficient for a higher $U_{y}$. In other words, the flow structures and their evolution show minor differences between the pitchinduced and $y$-directional free-stream-induced flow variations. For example, the flow without actuation at the moment of maximum lift at the angle of attack of $21^{\circ}$ shown in Figure $3 \mathrm{c}$ ) is very similar to the one with $U_{y}=1.5$ and the nominal actuation shown in Figure 3d). Despite their similarity, the actuation located at $x / c=0.2$ enforces flow reattachment, which disconnects the recirculation region, and the relevant length scales, such as separation bubble size and gaps between shed vortices, are smaller compared to the no-actuation case.

When the free-stream is subject to the time-varying disturbance, the aerodynamic forces exhibit dynamic trajectories uniquely characterized by disturbance parameters, disturbance amplitude and period (or Strouhal number and reduced frequency). As an example, Figure 4 shows the lift variation and flow-field snapshots for one disturbance cycle for the nominal flow case, i.e., open-loop actuation with the constant voltage of $2 \mathrm{kV}$. Compared to the static lift curve, the dynamic lift trajectory shows extended linearity with a much higher maximum lift and significant hysteresis. As shown in the snapshots, these features are related to the evolution of energetic vortex structures throughout the cycle, especially for the first half-cycle. The separation bubble initiated near the trailing edge develops into a massive recirculation region whose center first moves upstream and then transports downstream until it sheds around $t^{*} / T^{*}=0.5$. As detailed in the following section, the impact of these vortex activities on aerodynamic forces is sensitive to the disturbance conditions. In addition, the comparison between the case of this study and the one taken from Ref. 1 , which is based on different geometry and flow conditions, shows a 
noticeable similarity, suggesting that the findings in this study can provide insights into dynamic stall in different flow conditions.
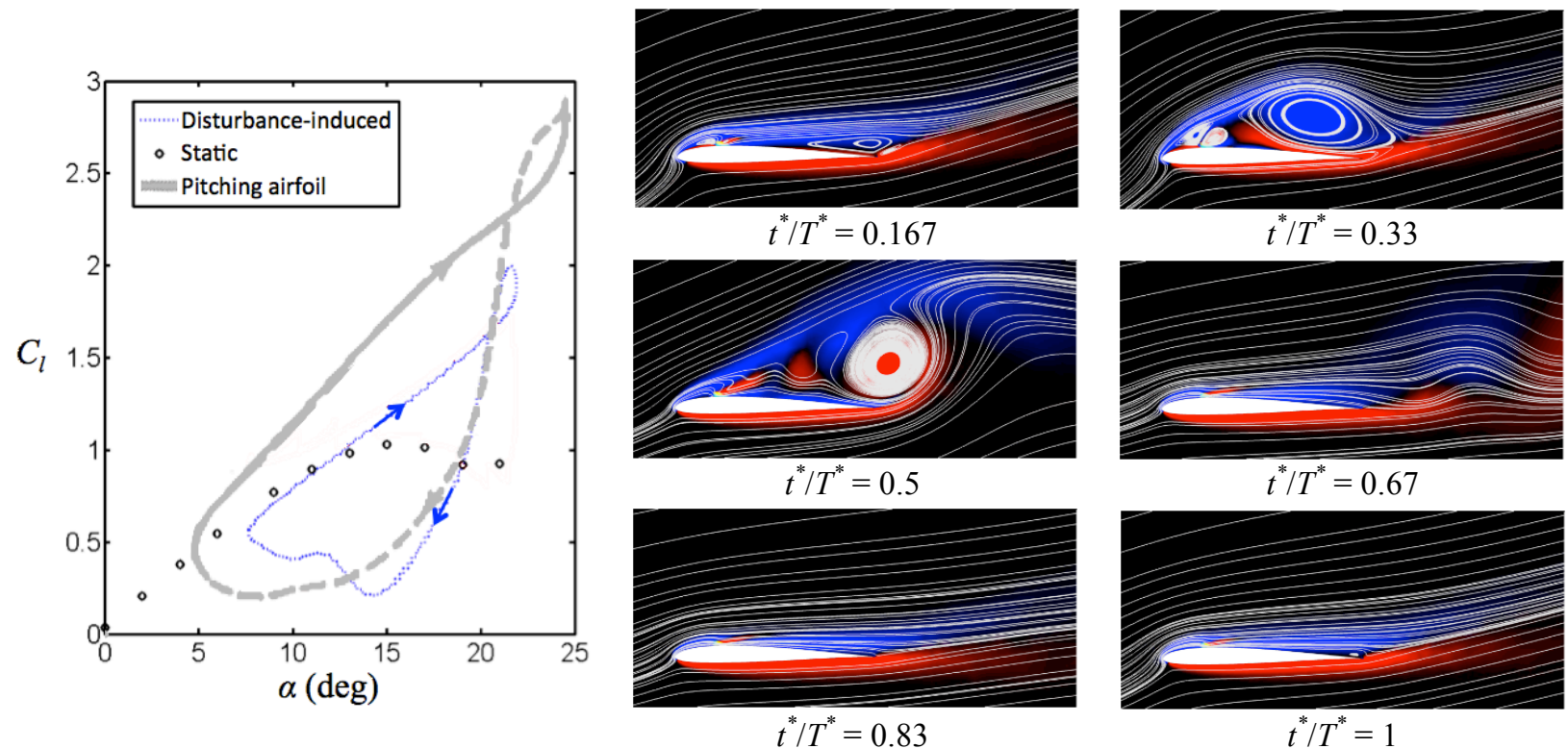

Figure 4 Static lift curve and disturbance-induced dynamic lift curves with open-loop control $\left(\alpha_{d}=0.5, T^{*}=\right.$ 10: $S t=0.13, k=0.31$ ) in comparison with the lift curve of a pitching airfoil adopted from Ref. 1 (NACA 0012 for $R e=O\left(10^{6}\right)$ at $k=0.25$ ), and streamlines and vorticity contours (blue: clockwise, red: counter-clockwise) at different time instants.

\section{B. Disturbance Regimes and Open-Loop and Closed-Loop Control}

The dynamic aerodynamic force responses can be viewed from two aspects: the impact of the changes in effective angle-of-attack due to disturbance and the impact of the evolving separation bubble, which can result in dynamic stall when the disturbance magnitude is large enough. Depending on the disturbance conditions, one can dominate the other, or both coexist, resulting in augmented complexities. Figure 5 shows a range of lift-drag relations in the same scale for different disturbance conditions. Each lift-drag profile corresponds to the variation of aerodynamic forces for one disturbance cycle for open-loop in solid black line and for closed-loop in solid red line. In addition, some characteristic contour lines are also shown in Figure 5 such as vortex shedding and multiharmonic oscillation limits, which are defined in the later part of this paper.

The lift-drag profile can represent the key flow features and their changes according to the disturbance parameters $S t$ and $k$. For Regime A, the relatively low disturbance amplitude result in small-magnitude harmonic oscillations in lift and drag regardless of the disturbance period. As the disturbance magnitude increases, the liftdrag profile deforms from a counter-clockwise circular profile into an ellipse in Regime B for small disturbance periods and into a triangular shape in Regime $\mathrm{E}$ for large periods. The major difference between Regime $\mathrm{B}$ and Regime $\mathrm{E}$ is whether the separation bubble evolves into shedding vortices (Regime B) or the flow is subject to static stall (Regime E). As a result, the lift-drag profiles in Regime E share some features of static stall shown in Figure 3. As the disturbance magnitude increases, the oscillation of separation bubble is amplified and as a result the lift-drag profile expands accordingly. Furthermore, as the growth and transport of the separation bubble become dominant, the second quadrant part of the circular (or elliptic) lift-drag profile moves to the first quadrant, resulting in the shape change in lift-drag relation into a collapsed or clock-wise profile in Regime C. When the disturbance frequency is reduced, however, this dynamic bubble expansion becomes less pronounced, resulting in smaller liftdrag profiles in Regime D. In addition, the lift-drag profiles in Regime D shows a figure 8 shape with highfrequency fluctuations caused by free vortex shedding in the wake. Finally, there exists some resonance frequency around $k=0.3$, where the disturbance peak is in phase with the evolution of separation bubble, resulting in the largest amplitude fluctuations in aerodynamic forces. The suggested categorization of disturbance conditions clearly shows the impact of disturbance-driven flow physics on aerodynamic forces. 


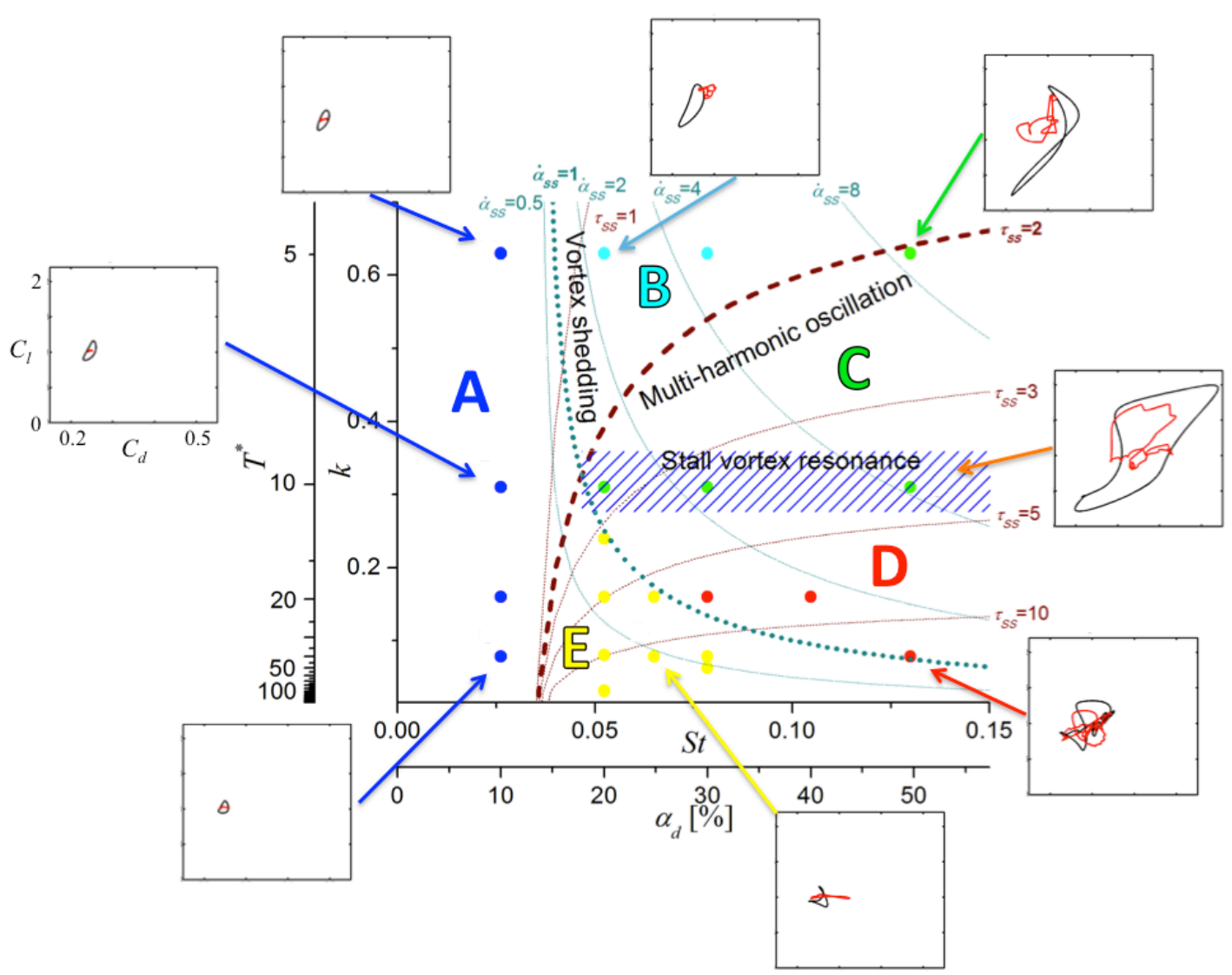

Figure 5 Disturbance regimes and open-loop (black) and closed-loop (red) lift-drag relations.

The closed-loop lift-drag relations show how effectively the lift variation can be mitigated by the control system and how drag changes meanwhile for different regimes. It is interesting that the lift mitigation results in the reduced drag fluctuations except Regime E. In addition, although the force fluctuations are less significant than Regime C, the control performance deteriorates in Regime $\mathrm{D}$, where both dynamic stall and free vortex shedding coexist.
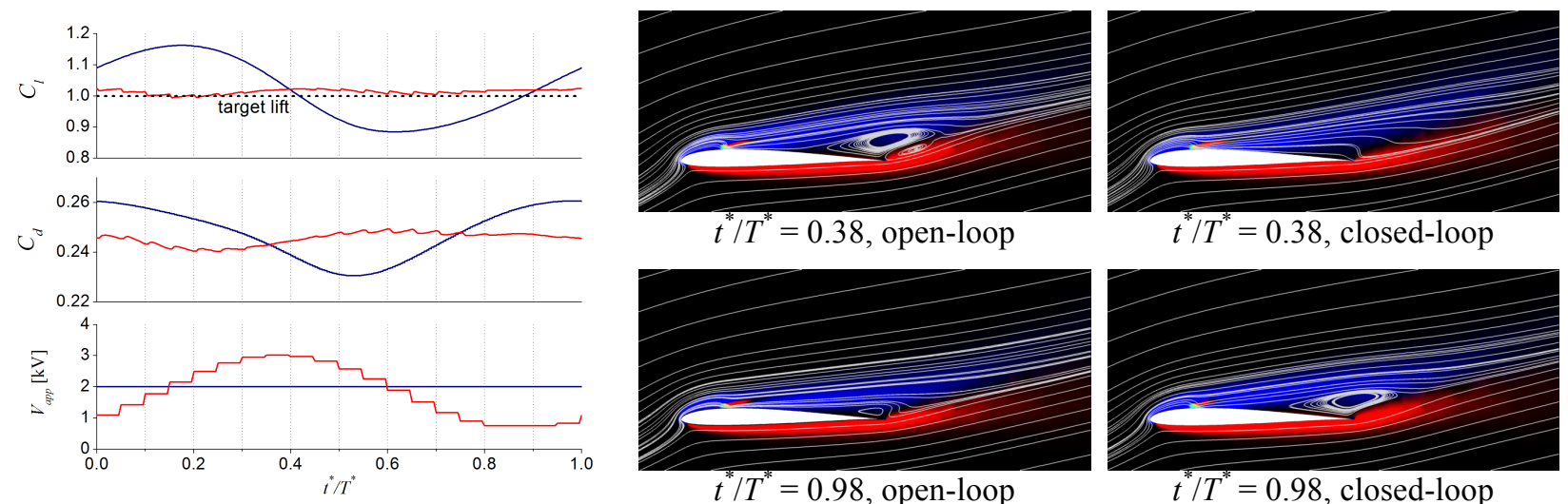

Figure 6 Aerodynamic forces and control for one disturbance cycle and flow-field snapshots (blue: clockwise, red: counter-clockwise vortices) for Regime $\mathrm{A}\left(\alpha_{d}=0.1, T^{*}=10\right)$.

Regime A: Single-harmonic oscillation

When the disturbance amplitude is relatively low, the aerodynamic force variation remains small, and the only noticeable impact of the disturbance on the flow field is resizing of the existing separation bubble in response to the free-stream oscillation. As a result, the open-loop lift and drag show harmonic variations as shown in Figure 6. The 
closed-loop voltage waveform is also close to a sinusoid with a phase-lag of about 0.2 with respect to the lift variation. The snapshots show that this voltage waveform advances the evolution of separation bubble about a half disturbance cycle. In other words, instead of suppressing the evolution of separation bubble, this phase offset in its evolution effectively stabilizes the lift variation.

\section{Regime B: Harmonic vortex shedding}

On the other hand, if the disturbance frequency becomes relatively high while the amplitude is moderate, the oscillating separation bubble evolves into shedding vortex structures. In Figure 7, although the open-loop lift variation is similar to the disturbance waveform, the sharp lift loss at $t^{*} / T^{*}=0.7$ coincides with the moment of vortex shedding as shown in the snapshot. During its evolution, the expanding separation bubble suggests that the disturbance phase-leads the growth of vortex, i.e., the angle-of-attack starts to decrease before the recirculating flow part reaches its maximum size. As a result, although the disturbance-driven vortex evolution is responsible for delayed stall and leads to the substantial lift loss, the decreasing angle-of-attack in the second half-cycle limits the growth of separation bubble, which becomes apparent when compared to the case of stall vortex resonance below.

The closed-loop voltage is, similarly to the lift waveform, close to the disturbance waveform with a little time lag, which brings the maximum voltage at the initial stage of the evolution/convection of separation vortex. As a result, the closed-loop system achieves an out-of-phase vortex evolution, i.e., synchronizes the possibly lowest lift moment with the moment of highest effective angle-of-attack and neutralizes the disturbance-induced angle-ofattach change using the advanced vortex shedding. In addition, the advanced vortex evolution due to the in-phase harmonic actuation substantially reduces the size of the shedding vortex.
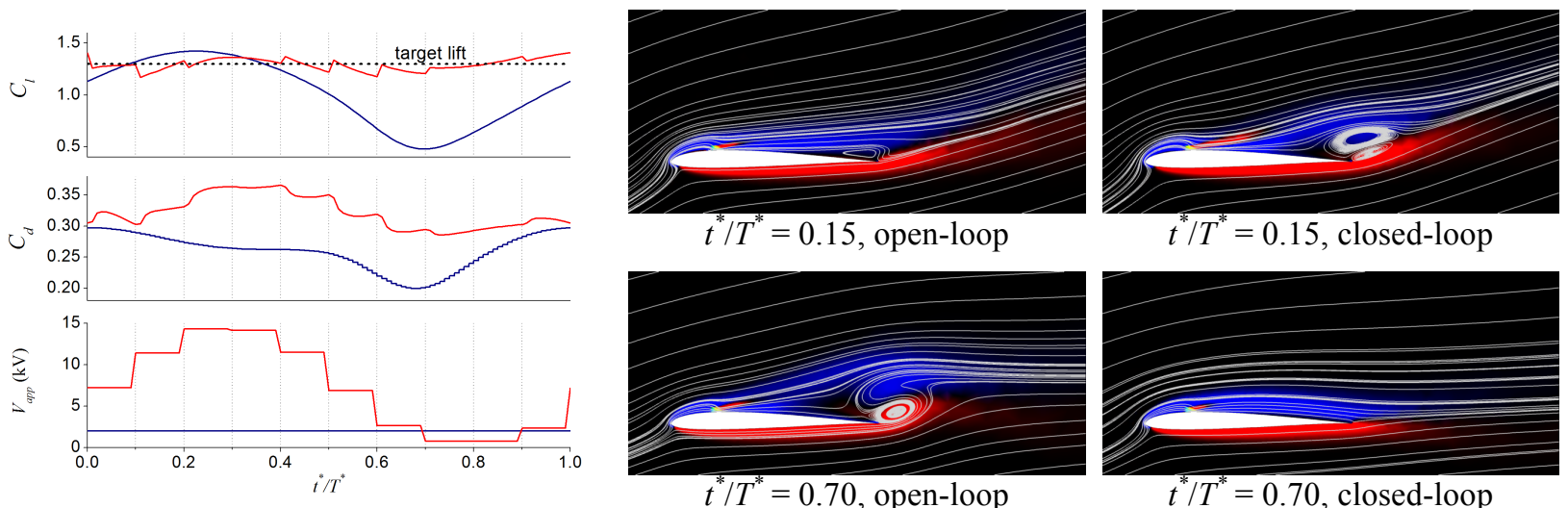

Figure 7 Aerodynamic forces and control for one disturbance cycle and flow-field snapshots (blue: clockwise, red: counter-clockwise vortices) for Regime $\mathrm{B}\left(\alpha_{d}=0.3, T^{*}=5\right)$.

\section{Regime C: Stall vortex dominance/resonance $(50 \%, 10 \mathrm{~T})$}

As the disturbance amplitude grows, the vortex activities within the separation bubble become more energetic. The open-loop lift history in Figure 8 shows non-harmonic oscillations including two lift dips. This multiharmonicity is related to the pronounced vortex activities during dynamic stall. Compared to the previous case of harmonic vortex shedding, the separation bubble has more time to expand to its maximum size before the disturbance speed reaches its maximum at $t^{*} / T^{*}=0.25$. After the maximum lift moment, the separation bubble evolves into a large vortex structures and is detached from the airfoil, resulting in the substantial lift drop.

As shown in the closed-loop lift history, the closed-loop voltage is maximum at about $t^{*} / T^{*}=0.35$, which is about 0.05 later than the lift peak, and in this case the lift fluctuation after $t^{*} / T^{*}=0.3$ is effectively suppressed. Here, the controller weakens and advances vortex growth and its shedding moment to about 0.3 , as shown in the snapshots. As a result, the moment of vortex shedding for the closed-loop case coincides with the maximum lift moment for the open-loop case, similarly to the harmonic vortex shedding case. On the other hand, the open-loop lift minimum due to the subsequent vortex shedding is prevented in the closed-loop case by the increased voltage, which increases the suction pressure near the leading edge as explained in the former study ${ }^{22}$. A similar maximum voltage even for higher lift fluctuations compared to single-harmonic vortex shedding can be explained in part by the lower target lift in this case. 


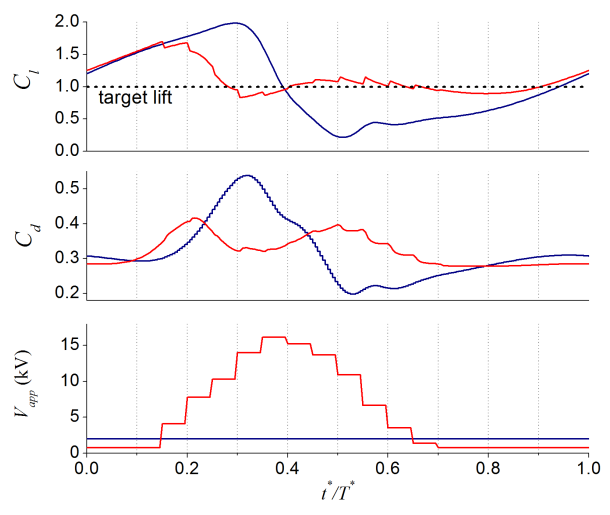

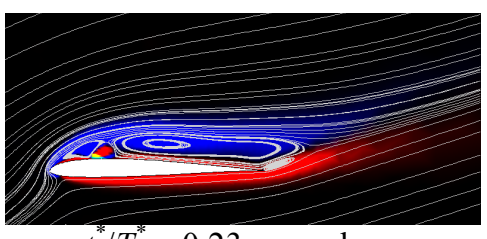

$t^{*} / T^{*}=0.23$, open-loop

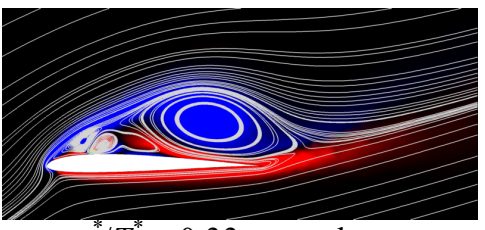

$t^{*} / T^{*}=0.33$, open-loop

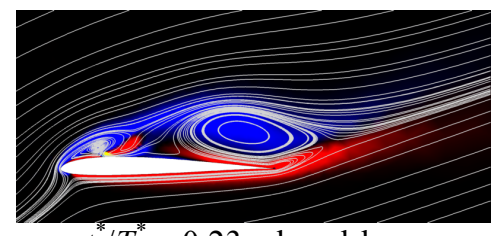

$t^{*} / T^{*}=0.23$, closed-loop

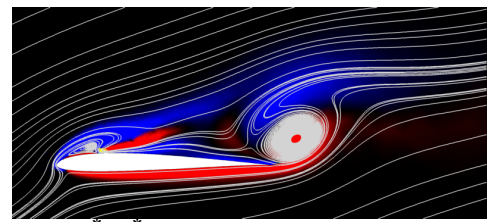

$t^{*} / T^{*}=0.33$, closed-loop

Figure 8 Aerodynamic forces and control for one disturbance cycle and flow-field snapshots (blue: clockwise, red: counter-clockwise vortices) for Regime $\mathrm{C}\left(\alpha_{d}=0.5, T^{*}=10\right)$.

Regime D: Mixed stall mode

A low disturbance frequency with sufficiently large amplitude accompanies a longer post-stall angle-of-attack interval, which provokes wake instability in addition to the unsteady evolution of separation bubble, resulting in mixed stall. As shown in Figure 9, compared to the case of stall vortex resonance, the magnitudes of force fluctuations are significantly reduced while the fluctuations due to free vortex shedding augment complexity. In addition, unlike other regimes, the separated flow region includes more vortex structures due to the slower and longer stall period. As a result, three dominant sources are responsible for the complex lift oscillations: disturbance dynamics, stall vortex development, and characteristic shedding frequency related to wake instability. Similar to the other cases mentioned above, the second half-cycle exhibits mostly attached flow and little variations in the flow structure. Compared to other regimes, the lift fluctuations in the mixed stall regime are subject to more difficulty in its control, mostly resulting in a poor control performance or controller failure. The closed-loop voltage whose maximum lies in the second half-cycle can marginally reduce the magnitude of lift fluctuation by slightly decreasing the wall pressure near the airfoil's leading edge.

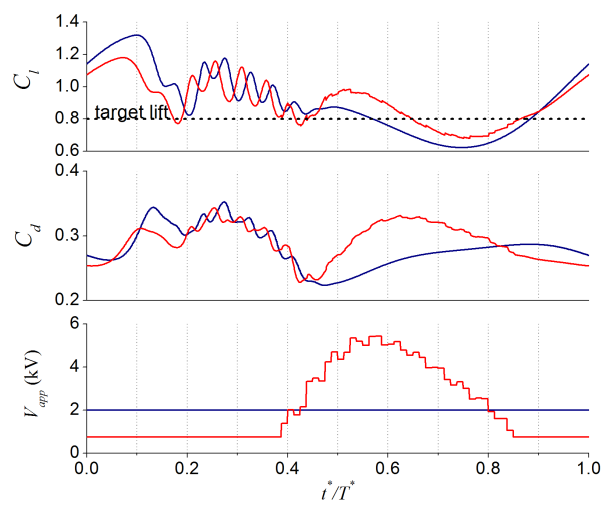

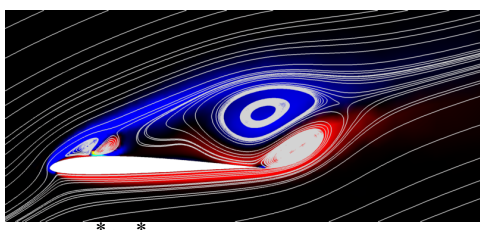

$t^{*} / T^{*}=0.15$, open-loop

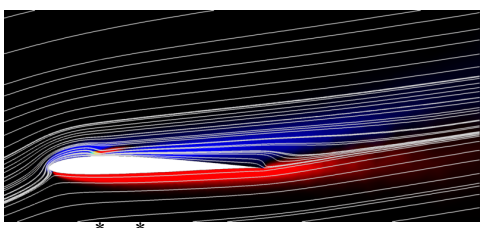

$t^{*} / T^{*}=0.58$, open-loop

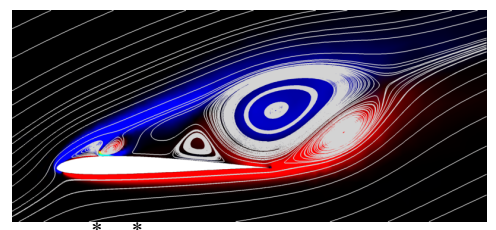

$t^{*} / T^{*}=0.15$, closed-loop

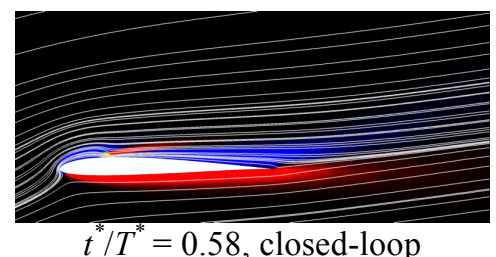

Figure 9 Aerodynamic forces and control for one disturbance cycle and flow-field snapshots (blue: clockwise, red: counter-clockwise vortices) for Regime $D\left(\alpha_{d}=0.5, T^{*}=40\right)$.

Regime E: Quasi-static stall

As the disturbance amplitude increases while the frequency is relatively low, the open-loop flow exhibits quasistatic lift stall: the stall angle for the lift loss shown in Figure 10 is close to the static stall angle. However, there is no delay in lift stall. As shown in the snapshots, the stall involves the stretch of separation bubble rather than vortex shedding, supporting the quasi-static nature of the flow dynamics in this regime. Note that the high curvature of the streamlines around the separation bubble is relieved at the end of stall due to the high viscous effects and relatively slow variations in free-stream. Unlike the cases with a higher disturbance magnitude, where the flow is detached from the entire suction side even with the actuation, the lift stall in this case accompanies only partial flow separation within the stretched separation bubble. 
The closed-loop voltage advances the growth of separation bubble, which, combined with the decrease in suction pressure due to the reduced voltage, can eliminate the initial lift peak, as shown in the snapshot at $t^{*} / T^{*}=0.09$. Then, the controller increases the actuation voltage for the rest of the first half-cycle to compensate for the lift loss due to the decreasing effective angle-of-attack. For the later part of the cycle, unlike the cases with higher disturbance frequencies, the lift dip caused by the reduced effective angle-of-attack is mitigated mainly by increasing the actuation voltage. It is interesting that the closed-loop voltage waveform is close to the reversal of the lift variation with a small phase-lead.
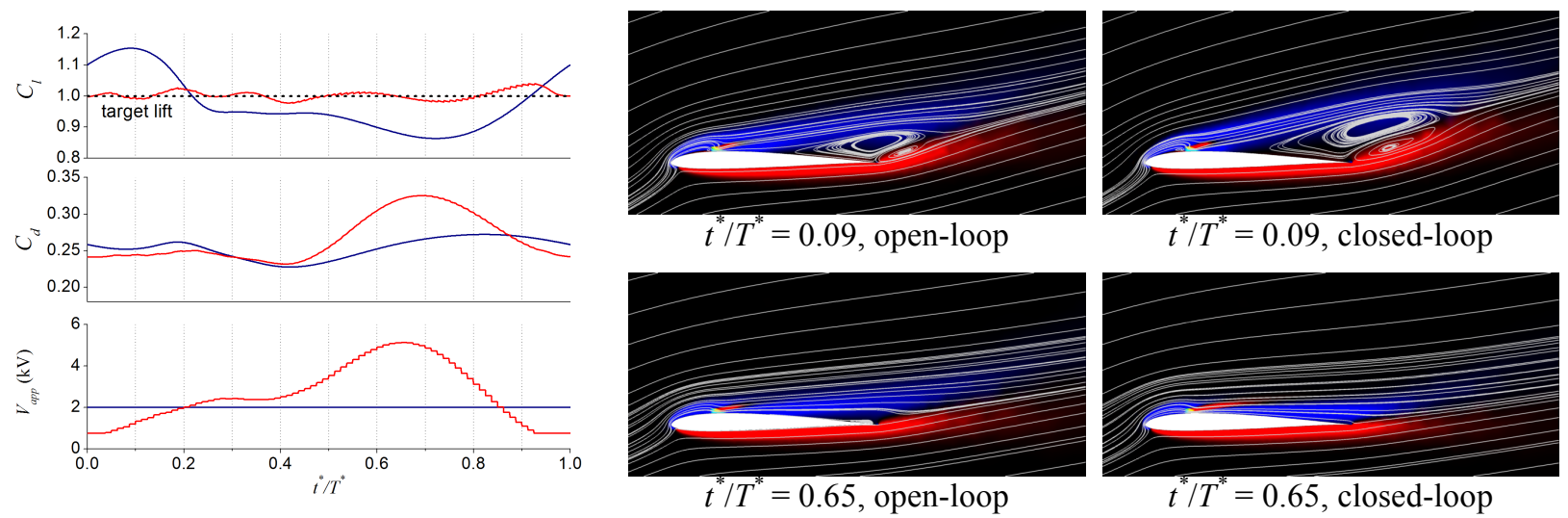

Figure 10 Aerodynamic forces and control for one disturbance cycle and flow-field snapshots (blue: clockwise, red: counter-clockwise vortices) for Regime $\mathrm{E}\left(\alpha_{d}=0.25, T^{*}=40\right)$.

\section{Disturbance Criteria: vortex shedding and multi-harmonic oscillation}

In many studies on dynamic stall, the variation of angle of attack around static stall angle is regarded as a key parameter that characterizes subsequent processes. Similarly, the initial rate of change in angle-of-attack due to disturbance, or disturbance slope, affects the evolution of stall vortex for large-amplitude disturbances. On the other hand, the evolution of separation bubble is subject to the intervening variation of angle of attack. For example, even with a high initial disturbance slope enough for developing a strong leading-edge vortex structure, its growth would be limited if a certain high angle-of-attack condition is not maintained during its expansion/convection phase.

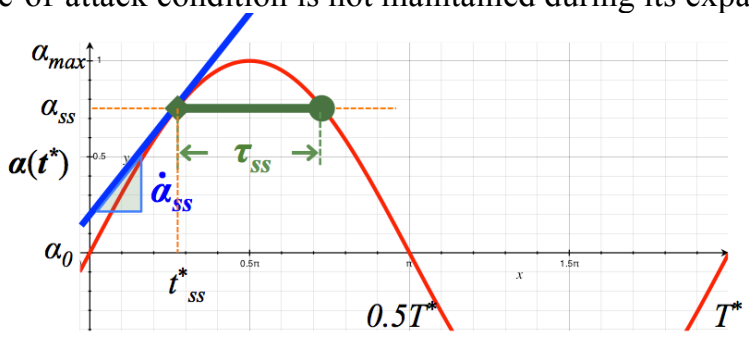

Figure 11 Time history of angle-of-attack due to sinusoidal disturbance and disturbance criteria.

Based on these observations, two disturbance parameters, the static stall slope $\dot{\alpha}_{s s}$ and the static stall interval $\tau_{S S}$, are used to represent the disturbance slope at the moment of static stall angle and the time duration of static stall, respectively. As shown in Figure 11, for a sinusoidal disturbance waveform, they can be defined using $S t$ and $k$ as follows.

$$
\begin{aligned}
\dot{\alpha}_{s s} & =\frac{\frac{2 k S t}{\alpha_{0}} \tan \alpha_{0} \cos \left(2 k t_{s s}^{*}\right)}{1+\tan ^{2} \alpha_{0}\left\{1+\frac{S t}{\alpha_{0}} \sin \left(2 k t_{s s}^{*}\right)\right\}^{2}}, \\
\tau_{s s} & =\frac{\pi}{2 k}-2 t_{s s}^{*}
\end{aligned}
$$


where $t_{s s}^{*}=\frac{1}{2 k} \arcsin \left[\frac{\alpha_{0}}{S t}\left(\tan \alpha_{0} \cdot \tan \alpha_{s s}-1\right)\right]$ is the moment of static stall onset. The contours based on these two variables are shown in Figure 5: cyan lines for the static stall slope and wine lines for the static stall interval. Here, two bold lines can be identified as the borders categorizing the disturbance regimes: the static stall slope of $1^{\circ}$ coincides with the onset of vortex shedding (cyan bold dotted line), whereas the static stall interval of 2 divides the single-harmonic and multi-harmonic responses (wine bold dashed line).

\section{Summary and Conclusions}

In this paper, the stabilization of aerodynamic forces under the influence of environmental gusts is considered. In particular, for a sinusoidal disturbance in vertical free-stream velocity, the open-loop and closed-loop responses of the flow-actuator system are investigated for an airfoil at the chord-based Reynolds number of 1000 . Here, the disturbance-induced flow physics and effective control mechanisms are detailed by defining characteristic regimes within the disturbance plane. The key findings can be summarized as follows.

1) The lift-drag trajectory of the flow-actuator system in response to sinusoidal disturbances identifies 6 disturbance regimes: in particular, vortex structure development, harmonicity in force oscillations, and stall mode are considered. The regimes are single-harmonic oscillation, quasi-static stall, harmonic vortex shedding, stall vortex dominance, stall vortex resonance, and mixed stall regimes.

2) The DBD actuator can mitigate the lift fluctuations in two ways: a) advancing and reducing the growth of separation bubble and $b$ ) directly changing the wall pressure distributions on the airfoil surface. Depending on the disturbance regime, the two mechanisms are used in different combinations. The current control system setup, however, exhibits performance degradation for the flows in the mixed stall regime, which accompanies high-frequency free vortex shedding in addition to the stall vortex evolution.

3) Two criteria based on the angle-of-attack variation and static stall angle are suggested to address the observed disturbance regimes. They correspond well to the boundaries of the onset of vortex shedding and multi-harmonicity in lift-drag trajectory.

In order to generalize the proposed categorization, more rigorous study is necessary to account for broader disturbance space and different flow conditions. However, the findings in this paper provide more clues to understanding the flow physics and control mechanisms effective for regulating aerodynamic forces under different disturbance conditions.

\section{References}

${ }^{1}$ Carr, L. W., "Progress in analysis and prediction of dynamic stall," J Aircraft, Vol. 25, No. 1, 1988, pp. 6-17.

${ }^{2}$ Visbal, M. R., "Numerical investigation of deep dynamic stall of a plunging airfoil," AIAA J, Vol. 49, No. 10, 2011, pp. 21522170 .

${ }^{3}$ Yarusevych, S., and Boutilier, M. S. H., "Vortex shedding of an airfoil at low Reynolds numbers," AIAA J, Vol. 49, No. 10, 2011, pp. 2221-2227.

${ }^{4} \mathrm{Yu}, \mathrm{M} . \mathrm{L} ., \mathrm{Hu}, \mathrm{H}$., and Wang, Z. J. "Experimental and numerical investigations on the asymmetric wake vortex structures around an oscillating airfoil," 50th AIAA Aerospace Sciences Meeting including the New Horizons Forum and Aerospace Exposition. Nashville, Tennessee, 2012.

${ }^{5}$ Mulleners, K., and Raffel, M., "The onset of dynamic stall revisited," Experiments in Fluids, Vol. 52, 2012, pp. 779-793.

${ }^{6}$ Greenblatt, D., and Wygnanski, I. J., "The control of flow separation by periodic excitation," Prog Aerosp Sci, Vol. 36, 2000, pp. 487-545.

${ }^{7}$ Cleaver, D. J., Wang, Z., Gursul, I., and Visbal, M. R., "Lift enhancement by means of small-amplitude airfoil oscillations at low Reynolds numbers," AIAA J, Vol. 49, No. 9, 2011, pp. 2018-2033.

${ }^{8}$ Fiebig, M., "Vortices and heat transfer," J Appl Math Mech, Vol. 77, No. 1, 1997, pp. 3-18.

${ }^{9}$ Zhang, P. F., Yan, B., Liu, A. B., and Wang, J. J., "Numerical simulation on plasma circulation control airfoil," AIAA J, Vol. 48, No. 10, 2010, pp. 2213-2226.

${ }^{10}$ Post, M. L., and Corke, T. C., "Separation control on high angle of attack airfoil using plasma actuators," AIAA J, Vol. 42, No. 11, 2004, pp. 2177-2184.

${ }^{11}$ Kopiev, V. F., Bityurin, V. A., Belyaev, I. V., Godin, S. M., Zaitsev, M. Y., Klimov, A. I., Kopiev, V. A., Moralev, I. A., and Ostrikov, N. N., "Jet noise control using the dielectric barrier discharge plasma actuators," Acoustical Physics, Vol. 58, No. 4, 2012, pp. 434-441.

${ }^{12}$ Chappell, S., Cai, Z., Zhang, X., and Angland, D. "Slat noise feedback control with a dielectric barrier discharge plasma actuator," 6th AIAA Flow Control Conference. New Orleans, Louisiana, 2012. 
${ }^{13}$ Grundmann, S., and Tropea, C., "Experimental damping of boundary-layer oscillations using DBD plasma actuators," Int $J$ Heat Fluid Fl, Vol. 30, 2009, pp. 394-402.

${ }^{14}$ Siegel, S., Cohen, K., and McLaughlin, T., "Numerical simulations of a feedback-controlled circular cylinder wake," AIAA J, Vol. 44, No. 6, 2006, pp. 1266-1276.

${ }^{15}$ Alam, M.-R., Liu, W., and Haller, G., "Closed-loop separation control: an analytic approach," Phys Fluids, Vol. $18,2006$.

${ }^{16}$ Kerstens, W., Pfeiffer, J., Williams, D., King, R., and Colonius, T., "Closed-loop control of lift for longitudinal gust suppression at low Reynolds numbers," AIAA J, Vol. 49, No. 8, 2011, pp. 1721-1728.

${ }^{17}$ Cho, Y.-C., and Shyy, W., "Nonlinearity identification and flow control for low-Reynolds number aerodynamics with unsteady free-stream," 50th AIAA Aerospace Sciences Meeting Including the New Horizons Forum and Aerospace Exposition, Nashville, Tennessee, 9-12 January, 2012.

${ }^{18}$ Santillo, M. A., and Bernstein, D. S., "Adaptive control based on retrospective cost optimization," J Guid Control Dynam, Vol. 33, No. 2, 2010, pp. 289-304.

${ }^{19}$ Cho, Y.-C., Hoagg, J. B., Bernstein, D. S., and Shyy, W., "Retrospective cost adaptive control of low-Reynolds number aerodynamics using a dielectric barrier discharge actuator," 40th Fluid Dynamics Conference and Exhibit, Chicago, IL, 28 June-1 July, 2010, AIAA 2010-4841.

${ }^{20}$ Kamakoti, R., Thakur, S., Wright, J., and Shyy, W., "Validation of a new parallel all-speed CFD code in a rule-based framework for multidisciplinary applications," 36th AIAA Fluid Dynamics Conference and Exhibit, San Francisco, CA, 5-8 June, 2006, AIAA 2006-3063.

${ }^{21}$ Shyy, W., Jayaraman, B., and Andersson, A., "Modeling of glow discharge-induced fluid dynamics," J Appl Phys, Vol. 92 , No. 11, 2002, pp. 6434-6443.

${ }^{22}$ Cho, Y.-C., and Shyy, W., "Adaptive flow control of low-Reynolds number aerodynamics using dielectric barrier discharge actuator," Prog Aerosp Sci, 2011.

${ }^{23}$ Skogestad, S., and Postlethwaite, I., Multivariable Feedback Control: Analysis and Design, New York, John Wiley \& Sons, 1996. 